\title{
Correlation of levels of alpha-fetoprotein in maternal serum and amniotic fluid in the rat
}

\author{
P. C. W. Lai, D. M. Hay* and F. L. Lorscheider \\ Divisions of Medical Physiology and ${ }^{*}$ Obstetrics and Gynaecology, Faculty of Medicine University of \\ Calgary, and Alberta Children's Hospital Research Centre, Calgary, Alberta, Canada
}

Alpha-fetoprotein (AFP) is a glycoprotein produced by the yolk sac and liver of the human conceptus and is found in maternal serum during pregnancy (see review by Lau \& Linkins, 1976). The source of AFP in the pregnant woman is thought to be the fetus. This conclusion is based on the correlation between AFP levels in cord and maternal serum at the time of delivery, and on the disappearance of AFP from the maternal circulation after parturition (Seppala \& Ruoslahti, 1972). In the rat, transplacental and trans-amniotic routes of AFP transfer have been suggested on the basis of radioisotopelabelled AFP tracer experiments (Sell \& Alexander, 1974), but quantitative data throughout gestation were not available. The objective of the present investigation was to determine whether a relationship exists between the levels of AFP in amniotic fluid and maternal serum throughout gestation.

Thirty-seven Sprague-Dawley rats (Canadian Breeding Labs, Constant, Quebec), 11-20 days pregnant, were used. Three or four rats were killed each day and the maternal serum and amniotic fluid were collected and assayed for AFP as previously described (Lai, Forrester, Hancock, Hay \& Lorscheider, 1976). The AFP value in amniotic fluid for each pregnant rat was derived from the mean value for amniotic fluid from five fetuses sampled at random from both uterine horns. Variation in amniotic fluid AFP concentration was evident among fetuses in the same pregnant rat. For all pregnant rats, the mean coefficient of variation was $21.9 \%$ with a range of $3.3 \%$ to $82 \%$. It can be seen from the paired maternal and amniotic fluid AFP levels that there is a significant correlation $(P<0.0001)$ between the concentration of AFP in amniotic fiuid and maternal serum (Text-fig. 1). The intercept of the regression line indicates that levels of AFP characteristic of pregnancy in the rat are manifest in amniotic fluid simultaneously or before they are detected in maternal serum. These findings suggest that the amniotic compartment may play a regulatory role in the equilibration of circulating AFP in both the fetal and maternal intravascular spaces and thus provide a conduit for the transfer of AFP from the fetus to the mother.

In contrast to the rat, the major route for human AFP transfer from the fetus to the mother is believed to be trans-placental. This interpretation is based on the study by Weiss, Macri, Robins \& Elligers (1976) which demonstrates that human maternal serum AFP rises quickly in response to

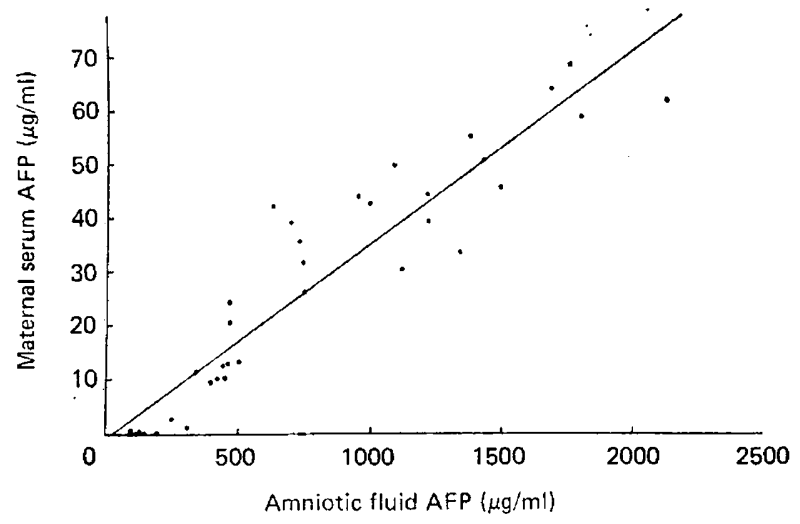

Text-fig. 1. Correlation of rat alpha-fetoprotein levels in maternal serum and amniotic fluid. Regression line: $y=-1.1045+0.0362 x ; n=37 ; r=0.9380 ; P<0.0001$. 
artificially induced acute fetal distress while there are no changes in amniotic fluid AFP concentration. The detailed mechanism and the regulation of transfer of AFP from the mammalian fetus to the mother remains to be elucidated. The result of the present study indicates that the amniotic compartment may play a significant role in regulating the fetal-maternal distribution of AFP in the rat.

This investigation was supported by the Medical Research Council of Canada, Grant No. MA 5292, and a grant from the Alberta Children's Hospital Research Foundation. P.C.W.L. is a recipient of an M.R.C. Studentship.

\section{References}

LaI, P.C.W., Forrester, P.I., Hancock, R.L., Hay, D.M. \& LORSCHEIDER, F.L. (1976) Rat alphafetoprotein: isolation, radioimmunoassay and fetal-maternal distribution during pregnancy. $J$. Reprod. Fert. 48, 1-8.

LAU, H.L. \& LINKINs, S.E. (1976) Alpha-fetoprotein. Am.J. Obstet. Gynec, 124, 533-554.

SkLL, S. \& AleXANDER, D. (1974) Rat alpha-fetoprotein. V. Catabolism and fetal-maternal distribution. $J$. natn. Cancer Inst. 52, 1483-1489.
SEPPala, M. \& Ruostahti, E. (1972) Radioimmunoassay of maternal serum alpha-fetoprotein during pregnancy and delivery. Am.J. Obstet. Gynec. 112, 208-212.

WeISS, R.R., MACri, J.N., Robins, J. \& Elligers, K.W. (1976) Maternal serum and amniotic fluid alphafetoprotein as a marker of acute fetal distress in a midtrimester abortion model. Obstet. Gynec., N.Y. 48, 718-722. 